\title{
Introduction to soft matter and neutron scattering
}

\author{
Julian Oberdisse* \\ Laboratoire Charles Coulomb (L2C), University of Montpellier, CNRS, Montpellier, France
}

\begin{abstract}
As an opening lecture to the French-Swedish neutron scattering school held in Uppsala ( $6^{\text {th }}$ to $9^{\text {th }}$ of December 2016), the basic concepts of both soft matter science and neutron scattering are introduced. Typical soft matter systems like self-assembled surfactants in water, microemulsions, (co-)polymers, and colloids are presented. It will be shown that widely different systems have a common underlying physics dominated by the thermal energy, with astonishing consequences on their statistical thermodynamics, and ultimately rheological properties - namely softness. In the second part, the fundamentals of neutron scattering techniques and in particular small-angle neutron scattering as a powerful method to characterize soft matter systems will be outlined.
\end{abstract}

\section{Introduction}

Soft condensed matter has grown into a rather wide and mature scientific discipline over the past 50 years, unifying different branches of physics, chemistry, and biology - with their interfacial disciplines physical chemistry, biophysics, etc. - into a common approach mainly characterized by the importance of entropy. There are many examples of initially independent fields, like surfactant science or liquid crystals, which have existed for more than one hundred years as a true 'nano'-science, or polymer physics, which really started with Staudinger and his macromolecular hypothesis between the world wars of the $20^{\text {th }}$ century. Some major progress was made by giants of science, sometimes rewarded - but not always - by a Nobel prize, like the ones of Staudinger ${ }^{\dagger}$, Ziegler and Natta in polymer chemistry ${ }^{\ddagger}$, as well as Flory in polymer physics and chemistry ${ }^{\S}$, or De Gennes for several contributions including polymer

\footnotetext{
${ }^{*}$ Corresponding author: julian.oberdisse@,umontpellier.fr

$\dagger$ The Nobel Prize in Chemistry 1953 was awarded to Hermann Staudinger "for his discoveries in the field of macromolecular chemistry".

$\$$ The Nobel Prize in Chemistry 1963 was awarded jointly to Karl Ziegler and Giulio Natta "for their discoveries in the field of the chemistry and technology of high polymers".

$\S$ The Nobel Prize in Chemistry 1974 was awarded to Paul J. Flory "for his fundamental achievements, both theoretical and experimental, in the physical chemistry of the macromolecules".
} 
physics $^{* *}$, a prize the late Sir Sam Edwards might have merited as well. The reason I mention these Nobel prize winners explicitly is that they enter in a particular resonance with the French-Swedish winter school held in Uppsala in December 2016, during the week of Nobel celebrations. The organizers had the brilliant idea to take us to the Nobel lectures in physics ${ }^{\dagger \dagger}$ (D.J. Thouless, M. Kosterlitz, D. Haldane) and chemistry (J.P. Sauvage, F. Stoddart, B. Feringa) in Stockholm, which was truly an extraordinary event.

In this short introduction - short with respect to entire textbooks available on the field of soft matter and neutron scattering [1,2], see also ref. [3, 4] from previous neutron schools , I will outline the fundamental concepts and properties of soft condensed matter, namely viscoelasticity, and its link to microstructure and thermal motion. These concepts are then illustrated with the main sub-disciplines of soft matter: from surfactants and microemulsions, to polymers and copolymers, colloids, and liquid crystals. I will then show how neutron scattering can be particularly appropriate to study such systems. Indeed, soft matter displays a wide range of different structures, as well as dynamical properties, and neutron scattering is a technique which is well-suited to unravel both their structure and dynamics, due to the unique properties of the neutron in terms of penetration power, wavelength, and energy. While different aspects of neutron scattering will be presented in detail in the following lectures, we restrict ourselves here to a discussion of small-angle scattering as a tool to characterize the distribution of both volumes and surfaces in space, with some hints to application of other scattering techniques to soft matter, like reflectometry, GISANS (grazing incidence small-angle neutron scattering), and QENS (quasi-elastic neutron scattering), all three techniques are also discussed in dedicated chapters in this volume.

\section{Fundamental concepts of soft condensed matter}

It will not surprise the reader to learn that the first and main property of soft condensed matter is its ... softness (i). But why is soft matter soft? Before going into microscopic detail, we need to turn to the branch of science which provides tools to characterize softness, called rheology, the science of flow. The basic rheological experiment - many different versions, time dependencies, and geometries exist, which we ignore here - is to exert a stress $\sigma$ (a force per unit surface) on the surface of a sample, and observe its deformation $\varepsilon$, or strain, given by the relative displacement of the surface, relative with respect to sample size. A weak stress inducing a large strain is characteristic of a soft material, and this is commonly described by a low elastic modulus $G=\sigma / \varepsilon$. A second property of many soft matter systems is that they can relax a stress they have undergone (ii), via more or less slow internal motions, introducing a characteristic relaxation time $\tau$. The latter is related to flow of the samples described by viscosity $\eta$, and $\eta$ equals $G \tau$ in the simplest case of a Maxwellian fluid [5]. Of course many complications exist, and these material 'constants' may be very complicated functions of time, or equivalently frequency, or history of deformation. But we may retain that soft samples are described by a modulus indicating elasticity, and by viscosity representative of flow: soft matter systems are thus usually viscoelastic. In practice, their linear rheological response is given by $G^{\prime}$ and $G^{\prime \prime}(\omega)$, where $\omega$ is the frequency $2 \pi f$ of a low-amplitude

\footnotetext{
** The Nobel Prize in Physics 1991 was awarded to Pierre-Gilles de Gennes "for discovering that methods developed for studying order phenomena in simple systems can be generalized to more complex forms of matter, in particular to liquid crystals and polymers".

$\Uparrow \dagger$ The Nobel Prize in Physics 2016 was divided, one half awarded to David J. Thouless, the other half jointly to F. Duncan M. Haldane and J. Michael Kosterlitz "for theoretical discoveries of topological phase transitions and topological phases of matter".

\# The Nobel Prize in Chemistry 2016 was awarded jointly to Jean-Pierre Sauvage, Sir J. Fraser

Stoddart and Bernard L. Feringa "for the design and synthesis of molecular machines".
} 
oscillatory shear experiment, or equivalently their Fourier transform $G(t)$. The latter is very useful, as it allows to discriminate solid from liquid behavior, $G(t)$ tending towards zero for liquids - if one waits long enough, illustrating that viscoelasticity is also a question of characteristic relaxation time. The poor person studying the bitumen drop experiment might very well be convinced to look at a solid over the time of a $\mathrm{PhD}$ - there is one drop about every ten years ${ }^{\S \S}$.

The origin of the viscoelastic properties of soft matter systems reside in their microscopic structure. Indeed, stress is transmitted across the sample by the building blocks of the specific soft matter system under study. These may be interacting neighboring colloidal particles, i.e. nanoparticles, or polymer molecules, etc. The more of them there are, the more stress is transmitted, i.e. the modulus $\mathrm{G}$ is directly proportional to the density of microscopic objects $\mathrm{n}$, which is related to the characteristic size of objects $\xi$ by $n=1 / \xi^{3}$. The third fundamental property (iii) of soft matter is that these building blocks are very big with respect to atoms making up atomic systems, like e.g. metals. Of course the nature of the cohesive forces is different there, we just wish to underline the enormous difference in strength related to the different sizes of the building block of the material. A polymer molecule, for instance, can be made of tens or even hundreds of thousands of atoms. It may be concluded immediately that polymeric systems are thousands of times weaker than, say, metals, and softness is thus related to microstructure (as well as to details of interactions). Similar arguments hold for any soft matter systems discussed in this article. Colloids, e.g., cover a typical size range from about a nanometer (below one would talk of an atomic cluster), to a micron. This upper limit is somewhat magic, as it represents an upper bound for several phenomena typical for soft matter, and deserves discussion. First, too big particles with respect to the wavelength of visible light scatter light strongly: suspensions become opaque, because of multiple light scattering, unless special index-matching conditions are met. Moreover, sedimentation sets in for big particles, because they become too heavy. This brings us to the fourth key property, (iv) Brownian or thermal motion. Its microscopic origin is due to the many random collisions with the much smaller solvent (or any matrix) molecules, and it results in a thermal energy per object of approximately $\mathrm{kT}$ - the exact value being not important for comparison, and depending on the number of degrees of freedom, each contributing on average $1 / 2 \mathrm{kT}$. If particles are too big, gravitational energy may overcome thermal energy, and particles sediment (or cream, like oil droplets in milk, depending on relative density). To find the critical size of particles below which Brownian motion dominates, one may either argue in terms of gravitational energy for a height of $\mathrm{R}$ equaling the thermal energy, or by comparing the particle speed due to thermal diffusion over a length of $\mathrm{R}$ to the speed of sedimentation. The critical size is somewhat magically fixed, as comparing displacement of particles by shear to Brownian motion - the ratio is called the Péclet number - gives the same result. The outcome is one micron, which delimits the colloidal domain. The phenomenon of thermal motion which is fundamental to colloid science and soft matter in general is thus linked to microscopic properties, namely collisions with much smaller particles, molecules and atoms, and therefore microstructure and size. Let's add that sedimentation measurements and their analysis, as well as confirmation of Einstein's theory of Brownian motion in 1905 [6], led to the precise determination of the Avogadro number, and to the Nobel prize of Jean Perrin in $1926^{* * *}$. It is a noteworthy coincidence in time and place that Theodor Svedberg received the

\footnotetext{
$\$ \S \mathrm{https}: / /$ en.wikipedia.org/wiki/Pitch_drop_experiment

*** The Nobel Prize in Physics 1926 was awarded to Jean Baptiste Perrin "for his work on the discontinuous structure of matter, and especially for his discovery of sedimentation equilibrium".
} 
Noble Price in chemistry the same year for his work on dispersed systems at University of Uppsala $^{\dagger \dagger}$.

Coming back to softness, the alert reader might argue that it is not only the number density, but also the strength of the interaction between the building blocks which sets the modulus. Soft matter interaction potentials may be of very different nature: colloids, e.g., may interact through a DLVO potential [7] summing up electrostatic and van der Waals terms, whereas polymer chains may pull on anchor points or induce depletion interaction for entropic reasons, or surfactant molecules might stick together following the hydrophobic effect, or form layers which can possess bending rigidity. The wealth of soft matter science actually comes from the endless possibilities of combinations of these interactions, like polymer molecules with hydrophobic stickers linking micelles, which mutually interact through electrostatic interactions, and so on. However, they all have one thing in common: the typical energy scale of interactions is close to the thermal energy, kT. Coming back to rheology, this property provides us with an estimate of the modulus of soft matter systems: $\mathrm{G}=\mathrm{kT} / \xi^{3}$.

As we will not speak much about colloidal interactions in this introductory chapter, we would like to mention that nanoparticles are often hard matter (inorganic) objects, or possibly polymeric latex beads, which usually cannot be compressed. Then the excluded volume interaction needs to be taken into account, opening the road to the transdisciplinary chapter of the (colloidal) glass transition. As often with soft matter, intermediate states exist, like star polymers which may eventually interpenetrate, depending on their architecture, and density.

On the other hand, systems which will be discussed in detail are polymer chains, as they illustrate perfectly the importance of entropy. Due to their many internal degrees of freedom, individual polymer molecules are statistical systems by themselves. As there is no energetical cost involved with going from one conformation to another, they are virtually all energetically equivalent. Thermal energy then generates Brownian motion between the different states. This leads to structure being averaged over large configurational spaces to be explored by the systems, and to dynamical properties as transitions between different conformations. Such dynamics are in the focus of neutron studies. From a thermodynamic point of view, the free energy $\mathrm{F}=\mathrm{U}$ - TS is essentially given by the entropy term, as $\mathrm{U}$ is mostly the same for the different conformations. Ludwig Boltzmann taught us towards the end of the $19^{\text {th }}$ century how to link entropy $\mathrm{S}$ to configurations (here polymer conformations, denoted $\mathrm{W}$, but this holds for any system): $\mathrm{S}=\mathrm{k} \ln \mathrm{W}, \mathrm{k}$ being the famous Boltzmann constant. This is why soft matter systems are entropy-driven, making the exploration of configurational spaces rather than enthalpic ones a key endeavour of soft matter science.

It is not easy to characterize what soft matter really is in one sentence. We have tried here to summarize its main properties (i-iv), which may seem rather disconnected. However, the particular rheological properties, softness and viscoelasticity, are linked to microscopic features common to all systems, which are the importance of entropy, object size and type of interactions - all of them setting the scale of transmitted constraints, as well as the reversibility of links between objects.

The outline of this chapter is the following: various soft matter systems will be introduced, starting with surfactant self-assembly and microemulsions. We then go to polymers, colloids and aggregates, and finally nanocomposite systems. In the second part, the fundamental

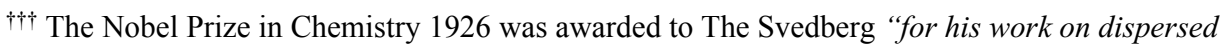
systems", namely in physical and colloid chemistry, and the development of the ultracentrifuge. 
scattering process of neutrons is introduced, and then discussed for the technique of small angle scattering, with special attention to applications for soft matter science.

\section{Physical properties of soft matter systems}

\subsection{Self-assembled surfactant systems and microemulsions}

Self-assembly. Surfactant molecules are amphiphilic molecules, i.e. they possess a hydrophobic and a hydrophilic moiety, which are usually an aliphatic chain (or several chains), and a hydrophilic head group, respectively. According to the nature of the head group, different classes exist, such as ionic and non-ionic surfactant molecules. The imagination and capacities of organic chemists being virtually without limit, many different types of molecules have been synthesized, including zwitterionic surfactants, double chain, or double (called gemini) or multiple head groups. There are two interrelated key properties of these molecules. On the microscopic side, they tend to self-assemble in a selective solvent, here usually water. The hydrophobic parts cluster together to avoid contact with water, and the hydrophilic parts decorate the interface towards water. The simplest aggregate is the micelle, which usually contains some tens, up to about a hundred molecules. This aggregation is the result of a thermodynamic balance between the translational entropy of the molecules, which tends to have them dispersed, and the hydrophobic effect, and it takes place at a welldefined (temperature-dependent) concentration, the critical micellar concentration, or cmc. The second, macroscopic, property which gave its name to the molecule, is that they are surface-active: as one adds them to water, the air-water surface tension is reduced considerably. The surface tension curve plotted against concentration presents a kink, located at the cmc, demonstrating that microstructure and macroscopic properties are intimately related.

Micelles are globular objects of radius approximately given by the aliphatic chain length. It is important to recognize that this radius is actually the radius of curvature of the interface. The exact geometry depends on a second parameter, the head group interactions, which might favor more or less curved interfaces, as described by the packing parameter $\mathrm{p}$ defined by $\mathrm{p}=$ $\mathrm{V} / \mathrm{l} \sigma[8,9]$. Here $\mathrm{V}$ is the molecular volume, $\sigma$ the head group area resulting from their interactions, and 1 the extended chain length. The compromise between the two parameters might lead to more spherical or more ellipsoidal surfaces, i.e. the higher the packing parameter, the lower the spontaneous interfacial curvature. This concept can be used to understand the sequence of morphology of micellar phases (called $\mathrm{L}_{1}$ ) shown at the bottom of Figure 1: the most bulky head groups give the lowest packing parameter, thus the highest curvature, and globular micelles are obtained. By either adding cosurfactant molecules having a smaller head group (for non-ionics), or screening electrostatic head group repulsion by adding salt (for ionics), the head group area is decreased, the packing parameter is increased, the curvature thus decreased, and micelles (highly curved monolayers in the two principal directions) deform into ellipsoidal structures, and finally cylindrical or wormlike micelles, with the monolayers curved on average only in one direction. 


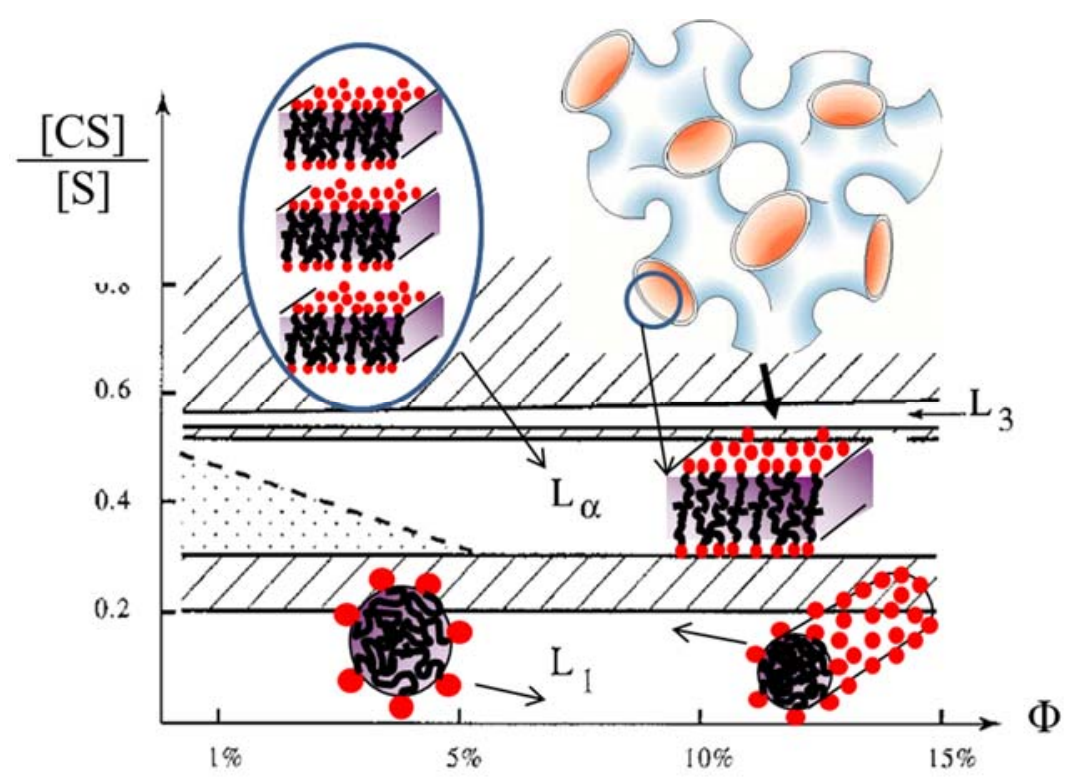

Fig. 1. Phase diagram of Triton X-100/octanol/water system. The $\mathrm{x}$-axis is the total amphiphile weight fraction, the $y$-axis represents the cosurfactant to surfactant ratio, [Oct]/[TX100]. The different surfactant aggregate morphologies belonging to the different phases are also indicated. Both $\mathrm{L}_{\alpha}$ and $\mathrm{L}_{3}$ are bilayer phases. Phase diagram first published in [10].

Decreasing the curvature even more leads to bilayer phases made of two on average flat monolayers sticking on each other, as illustrated in the middle part of Figure 1, for a Triton $\mathrm{X}-100 /$ octanol system. Micelles can thus be seen as a surfactant monolayer which is wrapped up on itself. The physics of surfactant systems is therefore given to a great extent by the bending properties of the monolayer, and of the bilayer for bilayer phases.

The formation of liquid crystalline phases is often encountered in soft condensed matter at high concentration, in particular with anisotropic molecules, or rods. As Lars Onsager yet another Nobel laureate, although not explicitly for phase transitions $\$ *$ - understood, this is due to the entropic optimization between individual rotation and collective translational motion. Above a critical concentration, the system prefers arranging into ordered materials, with positional or orientational order (described by the director) typical for solid crystals, while still maintaining its liquid aspects, thus forming liquid crystals. In surfactant or copolymer phases, the formation of liquid crystalline structures can be understood with the physics of monolayers outlined above [11-13]. In particular, the lamellar phase depicted in Figure 1 is already a (smectic A) liquid crystal with positional order in the direction perpendicular to the on average parallel and thus oriented bilayer planes. The physical mechanism underlying the sequence of liquid crystalline phases encountered at high concentrations is that surfactant molecules compete for hydration water of the head groups. As the concentration increases, there is less and less water, and therefore the packing parameter increases, triggering an evolution of aggregate morphology, from globular objects, to cylinders, to bilayers. This results in the formation of cubic micellar phases, followed by columnar liquid crystals. Then, as the monolayer curvature decreases further, branched cylinders are formed, which can arrange in bicontinuous cubic structures also referred to as

\footnotetext{
1t The Nobel Prize in Chemistry 1968 was awarded to Lars Onsager "for the discovery of the reciprocal relations bearing his name, which are fundamental for the thermodynamics of irreversible processes".
} 
"plumber's nightmare". The full zoology of liquid crystalline phases cannot be discussed here in detail, but for the sake of scattering analysis, we note that such ordered structures induce scattering peaks, which can be indexed and used to analyze the structure, as done for example with the famous isasomes [14-18].

In the following section, the most successful theoretical approach - the Helfrich Hamiltonian - to surfactant phases is discussed. It is also directly relevant to microemulsions, which are also presented. For didactical reasons, the introductory parts of a recent review on microemulsions [19] have been included in this article.

Bending properties and morphology of surfactant phases. The bending properties of surfactant monolayers were described in the 1970s by Helfrich et al [20], originally for lipid bilayers. These properties are commonly described by a series expansion of the bending energy for the lowest curvatures. The free energy dF needed to bend a piece of monolayer of surface dA can then be expressed in terms of the two principal curvatures $c_{1}$ and $c_{2}$, which are related to the principal radii of curvature by $c_{i}=1 / R_{i}$. The so-called Helfrich Hamiltonian of the bending free energy reads:

$$
d F=\left[\frac{\kappa}{2}\left(c_{1}+c_{1}-2 c_{0}\right)^{2}+\bar{\kappa} c_{1} c_{2}\right] d A
$$

The spontaneous curvature, $\mathrm{c}_{0}$, describes the state of lowest bending energy of the monolayer - note that notations with and without the factor 2 exist in the literature; here we chose to write the total spontaneous curvature as $2 \mathrm{c}_{0}$. Due to symmetry reasons, this term is zero for bilayers or balanced monolayers. Alternatively, the free energy can be formulated based on the total curvature $\mathrm{H}=\mathrm{c}_{1}+\mathrm{c}_{2}$, and the Gaussian curvature given by the product $\mathrm{K}$ $=\mathrm{c}_{1} \mathrm{c}_{2}$. The above expression for the bending energy serves as definition of two material constants of the monolayer, the bending moduli, $\kappa$ and $\bar{\kappa}$, which are sometimes called bending rigidity and saddle-splay modulus, respectively. Both moduli are in units of energy, i.e. do not refer to any length scale, which has the surprising consequence that the bending energy of a spherical shell as depicted in Figure 2 (with $\mathrm{c}_{0}=0$ ) is independent of its radius: $8 \pi(\kappa+\bar{\kappa} / 2)$.

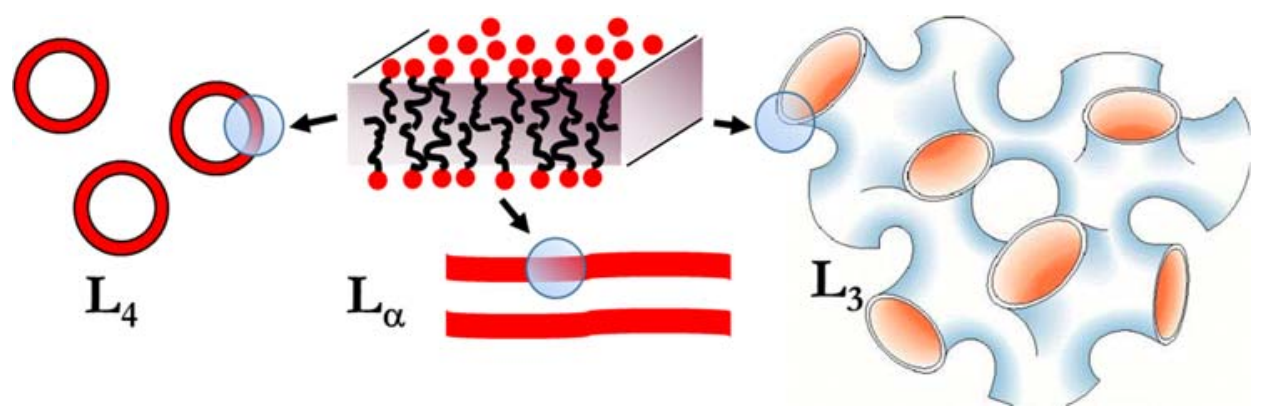

Fig. 2. Surfactant bilayer phases - vesicle, lamellar, and sponge phase.

The roles of $\kappa$ and $\bar{\kappa}$ are different. It can be shown with the Gauss-Bonnet theorem, that the second term in eq.(1), which is related to the Gaussian curvature, depends only on the topology of the surface, and in particular on the number of handles and independent pieces of membrane or monolayer [21]. As an example for these terms describing the topology of a membrane, one can think of a cup, which has one handle, and is topologically equivalent to a torus (e.g., like a doughnut). In order to have a handle (resp., many), a piece of matter needs to be curved in a particular way, and this is picked up by the Gauss-Bonnet theorem. The dependence on the Gaussian curvature is particularly obvious in the phase sequence of non 
ionic bilayer phases governed by the surfactant-cosurfactant ratio, where $\bar{\kappa}$ can be shown to trigger morphology changes from vesicular (called $\left.\mathrm{L}_{4}\right)$ [22], to lamellar $\left(\mathrm{L}_{\alpha}\right)$, and finally to sponge $\left(L_{3}\right)$ phases $[23,24]$, a sequence partially present in the phase diagram in Figure 1 , and shown in Figure 2. Concerning this last and most intriguing phase, it should be noted that there is a close relationship between the sponge phase and bicontinuous microemulsions to be discussed below [25]. Having understood the influence of $\bar{\kappa}$, it follows that for a fixed topology of any given structure, local topology-preserving bending fluctuations are entirely determined by $\kappa$, and can in principle be determined by dynamical studies [26, 27] .

Alternatively to eq.(1), the bending energy can also be expressed in terms of the deviation of the packing parameter (defined above) of the molecules from their spontaneous value $\mathrm{p}_{0}$. Indeed, if one integrates over the monolayer thickness $\lambda$, the packing parameter is found to be linked to the curvature in order to comply to complete surface coverage: $p=1+H \lambda+1 / 3$ $\mathrm{K} \lambda^{2}$ [28], as long as the topology remains unchanged. This covering relationship allows rewriting the Helfrich Hamiltonian as $1 / 2 \kappa^{*}\left(p-p_{0}\right)^{2}$, with $\kappa^{*}=2 \kappa+\bar{\kappa}$ [29]. One sees that monolayers adopting configurations close to spontaneous packing can only be stable with respect to bending if $\kappa^{*}$ is positive, i.e. if there is an energy penalty for spherical geometries.

\subsection{Microemulsions}

Microemulsions are surfactant systems which contain not only water, but also an immiscible oil. The role of the surfactant molecules is to allow a thermodynamically stable mixture of the two solvents. The canonical definition of microemulsions states that they are thermodynamically stable, isotropic, and liquid solutions of two immiscible fluids, typically oil and water, and surfactant [30]. Such systems present a low interfacial tension, combined with a high interfacial area between the immiscible fluids. They have many applications, basically everything which concerns mixing oily and aqueous matter, from food science to enhanced oil recovery. The properties of microemulsions are intimately related to their microstructure, and as this is a school on neutron scattering, you will be delighted to learn that neutron scattering is perfectly suited for the structural analysis of microemulsions. Besides strong and easily varied contrast, this is due to the essence of microemulsions: their structures are spatial distributions of domains of oil and water, respectively, delimited by a surfactant monolayer. Their geometry might be very complicated, but the measurement of the statistical properties of volumes and surfaces is exactly what neutrons, and in particular small-angle neutron scattering, can do for you. Moreover, by varying the contrast by deuteration, one can highlight either the bulk, or the interfacial layer, the latter being called film contrast. And last but not least, the Porod law gives a direct access to the specific (surfactant) surface, and thus the head group area $\sigma$. As already mentioned, the following paragraphs may also be found in a recent review [19].

The size and shape of the oil and water domains depend on the relative amount of oil and water, and surfactant. The usually encountered morphology for minority compounds, like a little oil with much water, are (discontinuous) oil droplets suspended in a continuous water phase, or vice versa. In approximately equal mixtures, both phases may become continuous, and bicontinuity will be discussed below. For more detailed information, we refer the reader to a general introduction to both physics and characterization of microemulsions proposed by Chevalier and Zemb [31], as well as to the bookchapter by Zemb [2].

Besides the volumes of the phases, microemulsion structure depends crucially on the quantity and properties of the surfactant. Indeed, the amount of the latter defines the available specific surface $\mathrm{S} / \mathrm{V}$, as all oil-water interfaces need to be covered for energetical reasons: $\mathrm{S} / \mathrm{V}$ is given by the product of surfactant concentration (number density) and head group area 
$\sigma$. Moreover, if we ignore solubility issues, the bending properties of the surfactant layer determine which type of morphology is accessible, and the strength of thermal fluctuation around these morphologies.

The existence of different phases in the phase diagram of microemulsions can thus be understood from the monolayer bending properties. A high curvature towards oil may set a typical droplet size, and the surfactant concentration the number of droplets. This defines how much oil can be solubilized, and emulsification failure with supernatant oil will in general be found for higher oil concentrations - forming what is called a Winsor I domain in the phase diagram, see Figure 3. There the number of phases of this domain are reported as $\underline{2}$, with the lower bar indicating the droplet phase below in the test tube. If the spontaneous curvature is of opposite sign, inverse systems with solubilized water in a continuous oil phase will be found - in case of emulsification failure as supernatant to an excess water phase, forming a Winsor II domain, noted here $\overline{2}$ in a similar way. Close to zero curvature, bicontinuous structures have been conjectured [32] and been proven to exist by NMR selfdiffusion and conductivity experiments [33-36]. In these Winsor III domains, both excess oil and water exist, denoted by the three phases " 3 " in Figure 3. If one now increases the amount of surfactant, more and more oil and water can be solubilized, up to a point where the excess phases disappear - forming a single bicontinuous microemulsion phase, denoted "1". The phase behavior described here in the curvature-surfactant phase plane for equal quantities of oil and water has the shape of a fish and is called the Kahlweit-Strey fish diagram [37, 38]. It is shown in Figure 3. It gives a direct illustration of the fact that the topology of the phases can be tuned and even inverted (the famous Shinoda cut) [39, 40], opening the road to chemical reactions either in droplets of either type used as microreactors, or at the (giant) interface of bicontinous microemulsions, benefitting of both available solubility channels in the samples[41, 42], also for decontamination[43, 44].

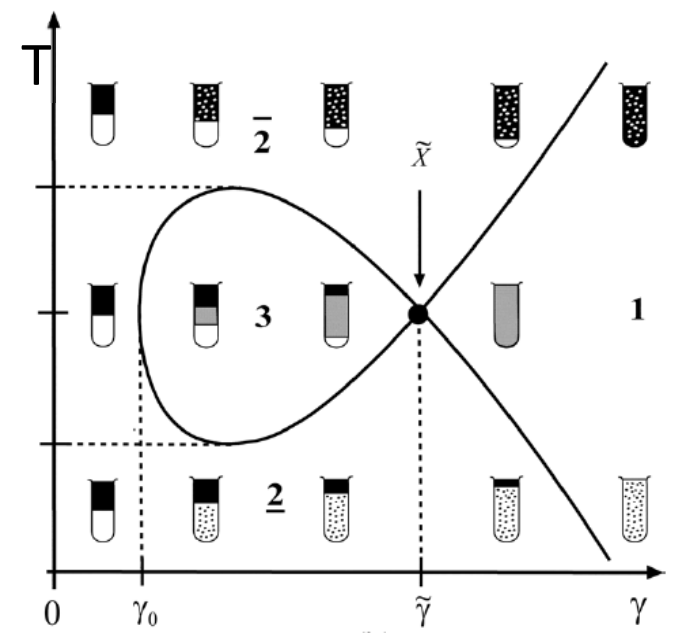

Fig. 3. Typical microemulsion phase diagram as a function of surfactant concentration and temperature. The Winsor phases are indicated, the numbers indicating the number of phases, forming the KahlweitStrey fish diagram, see text for details. Picture taken with permission from "Phase behaviour, Interfacial Tension and Microstructure of Microemulsions", T. Sottmann and C. Stubenrauch; pp. 1-47. Blackwell Publishing (Wiley-Blackwell) 2009.

\subsection{Polymers}

As already mentioned, it was recognized by Staudinger about one hundred years ago that polymers are macromolecules made of many small subunits called monomers. We start the 
discussion with a polymer molecule suspended in a solvent. Due to the many degrees of freedom, without any enthalpic preference, a polymer molecule is a statistical object in itself, possessing entropy, $\mathrm{S}=\mathrm{k} \ln \mathrm{W}$. The number of configurations depends on external constraints, like confinement, or stress on the molecule. If for instance we pull the chain ends apart, the number of configurations decreases, and so does $\mathrm{S}$, as the logarithm is a monotonic function. In absence of enthalpic contributions, the free energy $\mathrm{F}=-\mathrm{TS}$ thus increases, meaning that we have to provide work to pull the chain ends apart. This is illustrated in Figure 4, where two out of many possible chain conformations are drawn for an identical end-toend distance on the left and in the middle, whereas the stretched chain on the right has almost no degrees of freedom left. The corresponding force on the chain ends is thus of entropic origin, and there is an entropic spring constant describing this process. Not surprisingly, it is found to be proportional to $\mathrm{kT}$, as the thermal motion is at the origin of this elasticity. Together with the density argument outlined in section 2 , the modulus of a network of chains, called elastomer or rubber, is thus also given by $\mathrm{G}=\mathrm{kT} / \xi^{3}$, where the characteristic size $\xi$ now describes the distance between chain connections (called crosslinks, or possibly entanglements).
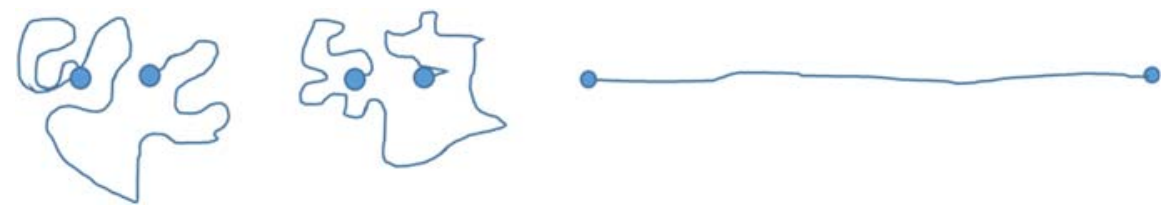

Fig. 4. Illustration of two polymer chains having the same end-to-end vector represented by the blue dots (left and middle). On the right, a stretched chain.

Coming back to individual chains, the distribution of chain ends can be described for an ideal chain, i.e. ignoring monomer interactions and excluded volume, by a Gaussian [45, 46], which is why these chains are also called Gaussian chains. The distribution function for the end-to-end vector $r$ of such a chain made of $\mathrm{N}$ segments of length $\mathrm{b}$ reads:

$$
P(r)=\left(\frac{3}{2 \pi N b^{2}}\right)^{3 / 2} \exp \left(-\frac{3 r^{2}}{2 N b^{2}}\right)
$$

The Gaussian distribution function can be derived from a random walk describing the polymer conformation, and it thus also describes unperturbed Brownian motion of particles, by treating the number of monomers $\mathrm{N}$ and the time as analogous quantities. If, however, the excluded volume of the monomers needs to be taken into account, then a self-avoiding random walk is appropriate. The difference between the two is that the self-avoiding chain is bigger due to the monomer repulsion. This is the case for particular solvents, called 'good solvents', whereas the ideal case is only found in so called $\Theta$-solvents, with reference to a specific temperature $\Theta$ where monomeric interactions are screened.

The typical size of a macromolecule is described by the average radius of gyration $\mathrm{R}_{\mathrm{g}}$, which is also the quantity directly measured by SANS, see the next section. The scaling laws of this radius with the number of monomers are characteristic: $\mathrm{R}_{\mathrm{g}}$ is proportional to $\mathrm{N}^{1 / 2}$, and $\mathrm{N}^{3 / 5}$, for Gaussian and self-avoiding chains, respectively. The exponent is also measurable by SANS, it appears as the inverse of the average fractal dimension of these objects, cf. below. One might add that a single macromolecule will of course never possess exactly the measured radius of gyration: it is the strength of scattering techniques to average over the many molecules evolving in the beam. The type of average, however, depends on polydispersity, which is why some care is necessary for data analysis. On the other hand, the situation is analogous to measuring the temperature in a room: usually no one is interested in the speed 
of all the air atoms, we are all happy with a single number, $\mathrm{T}$, describing the average energy of all of them.

In order to get an idea of the virtually infinite number of possible conformations of large molecules, we take the example of a long molecule, say one carrying 10000 monomers. If there are three possible bond angles between two of them, and if we ignore excluded volume, then there are $3^{10000}$ possibilities - an enormous number, approximately $10^{5000}$. If it takes one picosecond to explore one configuration, then it takes much much much longer than the age of the universe (!) to explore all individual conformations of a molecule, between completely stretched, and a small coil. Also, the ratio in size between the two extremes is roughly a factor of one hundred, and one can try to imagine all the intermediate possibilities. Luckily, the Gaussian distribution in eq.(2) also tell us that most conformations correspond to approximately the same typical size of the random coil given by $\mathrm{Nb}^{2}$, leading to a rather well defined average value.

The exploration of the many possible configurations of a large molecule immediately introduces dynamics occurring as the transitions of one microstate to another. Measurement of dynamics with neutrons which are an ideally suited probe for soft matter due to the available q- and energy-range, will be described in the lecture by Jean-Marc Zanotti [26]. We will thus not go into detail here, but would like to mention at least the fundamental concepts of the broad field of polymer dynamics. First, polymers as individual molecules undergo thermal motion, typically described by Rouse and Zimm modes [45]. Experimentally, these motions lead to a loss of correlation with respect to some initial state with time, and correlation functions are exactly what is measured by neutrons with quasi-elastic methods, called QENS [47-50]. With these methods, it is possible to either follow individual protons using incoherent scattering, or entire chains if appropriately labelled, using coherent measurements. In dense polymer systems, like melts, i.e. in absence of solvent, polymer molecules are constrained by their neighboring molecules, as illustrated in Figure 5. It was recognized by De Gennes in the early 1970s that they could only move away from their current conformation by moving along the chain direction. This peculiar motion is called reptation, in analogy with the motion of reptiles, like snakes. Reptation can be imagined as the back- and-forth motion of a chain in a tube formed by the neighboring chains. This generates a very particular 'tube-escape' dynamics, and the tube model has been highly successful in describing microscopic polymer dynamics and rheology. Interestingly, the link between the last two a priori independent measurements is given by the underlying relaxation mechanism of the polymer chains, which are also stress carrying entities. The 'softness' and slow relaxation of soft condensed matter is thus once again based on thermal motion governing interactions and reorganization of large objects, over large length scales.
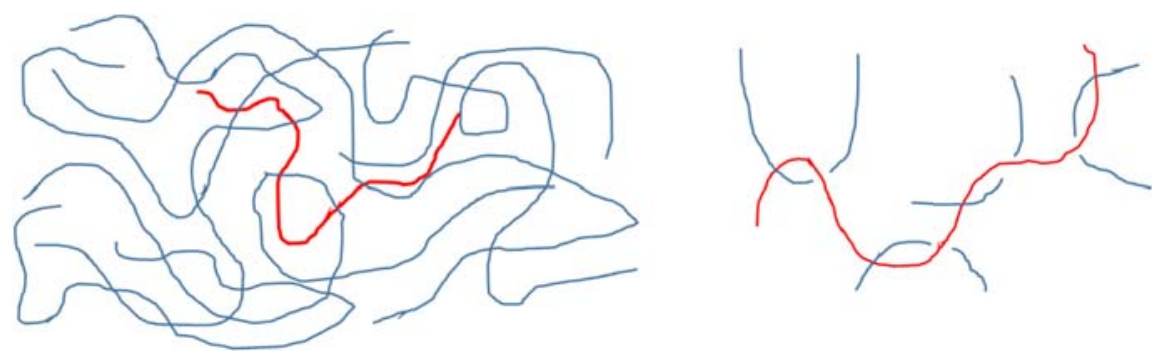

Fig. 5. On the left, illustration of a chain in a melt of chains. On the right, illustration of constrained topology of the red chain by the neighboring chains forming the tube.

As a last comment in this section, we may note that polymer physics did not stop with linear chains, which is mainly the chemists" "fault". Other architectures, and other 
interactions, exist, of arbitrary complexity, like the already mentioned copolymers, but this is outside the scope of this chapter. The lectures by Combet [51] and Rubatat [52] provide an idea of the infinite possibilities.

\subsection{Colloids and aggregates}

Colloids are another major building block of soft condensed matter. The word is of Greek origin, related to stickiness. Today one speaks mostly of nanoparticles, but they have been used spontaneously for a long time by mankind. Gold particles studied by M. Faraday in the $19^{\text {th }}$ century are generally cited as the first scientific approach to colloids.

In spite of the many funny shapes nanoparticles may have, we will restrain ourselves here to the discussion of spherical and mostly monodisperse nanoparticles. The dispersion state of nanoparticles suspended in a solvent depends on the interaction between the particles. The translational (or mixing) entropy of the particles tends to a random dispersion, whereas attractive interactions may induce aggregation and phase separation, and repulsive interactions may structure the suspension, and stabilize it. The most important interparticle interactions are excluded volume interactions of the particles themselves, followed by long range electrostatic interactions for charged particles [7], or steric repulsion due protective layers formed by molecules adsorbed or attached (grafted) to the particle surface. On the other hand, attractive van der Waals forces are always present but short-ranged, and depletion forces due to exclusion of smaller particles - they create osmotic pressure - may have intermediate ranges given by the size of the excluded object. Depending on particle concentration, very different phenomena might occur, like the colloidal glass transition at high concentration $[53,54]$, the discussion of which is however outside the scope of this chapter.

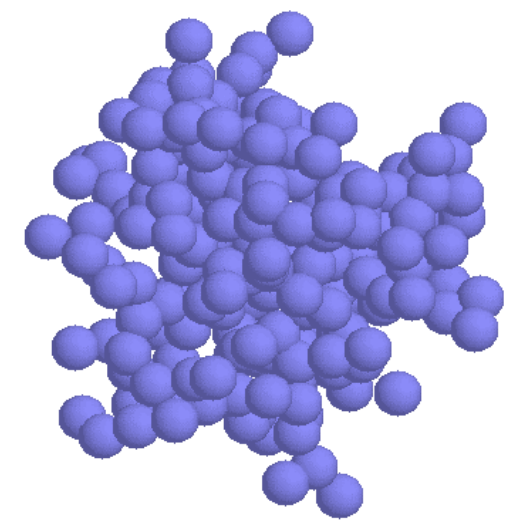

Fig. 6. Illustration of a simulated aggregate of colloidal spheres.

Colloidal aggregates as illustrated in Figure 6 may form due to any of the abovementioned interactions. They are encountered as soon as colloidal stability is weakened, and their shapes and in particular fractal dimensions have been extensively discussed in the literature. Some thirty years ago, there was a major hype on relating fractal dimensions to the type of diffusion process leading to collisions and 'reaction' in the sense of stickiness, like reaction or diffusion-limited aggregation (RLA or DLA, resp.), or similarly clusteraggregation on a larger scale. For the purpose of the present chapter, we dare the comment that the hope of universality was somewhat disappointed. It is true, however, that fractal dimensions are the appropriate property for certain classes of aggregates, and that the latter can be easily accessed by scattering methods as explained below. Finally, aggregates are of 
major importance for some complex applications of soft matter, like polymer nanocomposites, as outlined in the following section.

\subsection{Introducing complexity by combining soft matter systems}

In the above paragraphs, we have outlined the most fundamental properties of soft matter, with special emphasis on surfactant systems and microemulsions, polymers, colloids, and colloidal aggregates. As already indicated in some cases, these properties can be coupled, and mixed systems are constantly invented. Needless to say, they all cannot be presented here, but some may be mentioned as examples: copolymers and their mixtures with micellar systems [55-57], forming sometimes complex colloids [58-66], or polyelectrolyte complexes [67-69], copolymers added to microemulsions [70, 71], and of course many systems of biophysical [72] or agronomical interest [73, 74]. Moreover, polymer molecules may have interesting thermodynamic properties, like microgel particles displaying a volume phase transition [75-77]. Colloids may form structures, but they may also be studied for their individual motion, like self-propelled particles [78]. Other sub-disciplines of soft condensed matter have also been excluded from this review focused on microscopic aspects, like wetting, or textures of thermotropic liquid crystals.

Polymer nanocomposites are a famous application of both colloids and polymers. These are materials made of hard and usually inorganic nanoparticles embedded in a soft polymer matrix. Car tires are famous examples, with carbon black or silica playing the role of the filler nanoparticles. Their role is to reinforce the polymer, in order to decrease abrasion and increase stiffness and strength, while keeping wet skid properties - the tire sticking to the road - as good as possible, without compromising the lowest possible rolling resistance. The latter is responsible for the about $20 \%$ of the fuel consumption, and thus $\mathrm{CO}_{2}$ production. From a fundamental point of view, many scientific questions arise, related to the rheology of the material, and its non-linear mechanical properties. It is thus important to study both the microscopic structure and the dynamics of such systems, in order to understand 'more useful' properties. The microstructure includes chain conformation and filler dispersion, and the dynamics can be studied on any scale from atoms, to molecules, to nanoparticles, and finally to NP aggregates and networks. As you may doubt, neutron (and also x-ray) scattering is well suitable for such studies. For further reading, the reader is referred to our recent work on model [79-83] and industrial [84-88] systems, including dynamics [89, 90], as well as reviews [91, 92].

After having reviewed the main disciplines and systems of soft condensed matter, we now turn to neutron scattering, and in particular structural characterization by SANS. It is recalled that more details can be found in the following chapters, which corresponds to the lectures given in this school: after an introduction to the properties of neutrons by T. Ederth [93], a variety of applications of neutrons to soft matter systems is presented: Jérôme Combet focuses on polyelectrolytes and small-angle scattering [51], Camille Loupiac et al discuss neutron imaging and tomography applied to food science [94], and Laurent Rubatat introduces block copolymer electrolytes for fuel cells [52]. Then three chapters are devoted to dynamics and two surface techniques, respectively. Jean-Marc Zanotti et al present inelastic and quasi-elastic scattering applied to soft matter [26]. Finally, Fabrice Cousin and Alexis Chennevière discuss neutron reflectivity aiming at density profiles perpendicular to surfaces [95], and Max Wolff, the main organizer of this winter school with Fabrice Cousin, introduces grazing incidence scattering capable of revealing in-surface structures[96].

\section{Introduction to neutron scattering and SANS}

Neutrons as a probe to explore the structure and dynamics will be presented in detail in the chapter of Thomas Ederth [93]. They are highly suitable for soft matter studies, primarily for 
four reasons. First, they have a high penetration power of matter, which enables one to study thick samples - typically in the millimeter range, which is a lot more convenient than, say, one hundred nanometer slices necessary for TEM for instance. This does not only limit the introduction of artefacts with sample preparation, but also favors the measurement of true averages over large and thick samples. Secondly, the wavelength of cold neutrons is in the 5 to $20 \AA$ range, and the scattering wave vector $\mathrm{q}$ is thus appropriate for the study of nanostructures. Here one also understands immediately that the wavelength of typically $10 \AA$ is still a bit too small as compared to large soft matter structures, which may reach up to thousands of Ångström, with the consequence that one needs to measure at small angles (typically well below a few degrees) in order to characterize these structures [96]. Thirdly, the energy of the neutron is comparable to the thermal energy kT, which we have seen to be characteristic for soft matter systems. It can thus efficiently exchange energy with the sample, and the measurement of these energy changes in inelastic or quasi-elastic scattering experiments give information on the system's dynamics [26]. Finally, the nuclear interaction between the neutrons and the nuclei may be very different for isotopes, and in particular for protons and deuterium. This leads to the possibility of isotopic substitution, i.e. the introduction or modification of scattering contrast via the chemistry. As water is ubiquitous in soft matter studies, the (possibly partial) replacement of $\mathrm{H}_{2} \mathrm{O}$ by $\mathrm{D}_{2} \mathrm{O}$ can be used to highlight the scattering contributions of parts of a sample, giving access to otherwise hidden information. Another famous example to be discussed below is the structure of polymer chains in a melt.

\subsection{Basic scattering process of neutrons}

When cold or thermal neutrons interact with matter, their typical wavelength is in the $\AA$ to $\mathrm{nm}$ range, whereas nuclear interactions take place at a femtometer scale. As a result of this point-like interaction, a spherical secondary neutron wave is created, centered at the position of the scattering nucleus. In case of elastic interactions, this spherical wave has the same wavelength and energy as the incoming wave, but spreads in all directions of space, contrarily to the incoming wave, which is directed towards the beamstop, see Figure 7.

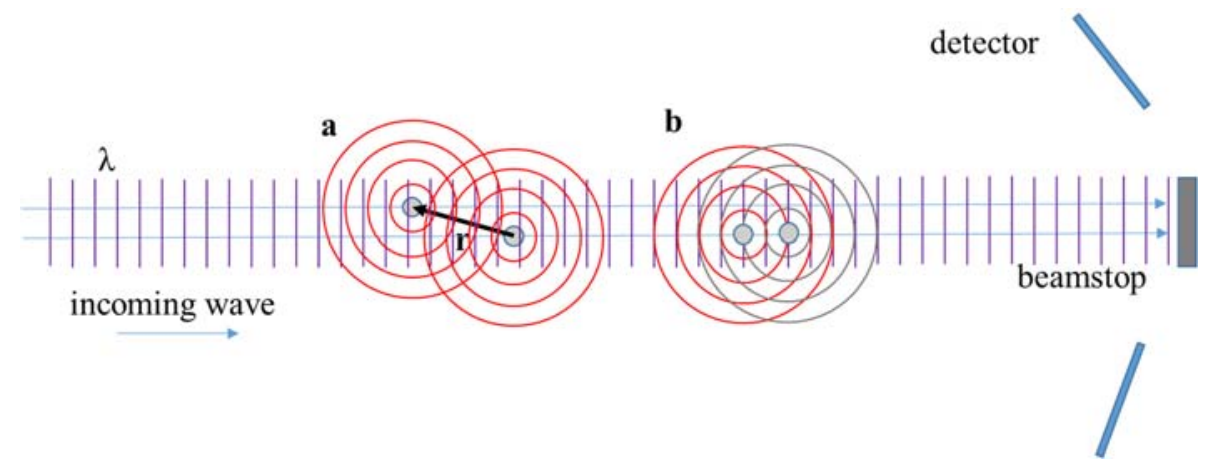

Fig. 7. (a) Illustration of spherical secondary waves produced by two scattering centers located at a distance $r$. Their interference may be measured on a detector. The incoming wave of wavelength $\lambda$ comes from the left, and the forward direction is to the right. Note that the secondary waves are in phase in the forward direction $(\Delta \Phi=0)$ independently of $\mathrm{r}$ - a particular configuration $\mathrm{r}$ has been chosen to illustrate this in (b), but it is true for all $\mathrm{r}$.

By measuring a non-vanishing neutron flux outside the beamstop, we can thus conclude that there must be at least one atom in the beam! Moreover, the amplitude of the scattered wave is proportional to the scattering power of the nucleus. The latter has the unit of length, 
and is called the (coherent) scattering length of this nucleus $i$, usually denoted $b_{i}$. The situation becomes more interesting for two atoms, located at a distance $r$, also shown in Figure 7. Now the two secondary waves can interfere, i.e. their amplitudes add up, and the result of this addition depends on their phase shift. The latter depends on the distance between the atoms $\mathrm{r}$, and on the direction in which the interference is measured. This direction is described by the scattering angle $\Theta$, which is usually defined as the angle between the primary beam and the scattering direction. A straightforward calculation shows that the norm of the scattering vector $\mathrm{q}$ is given by:

$$
\mathrm{q}=4 \pi / \lambda \sin (\theta / 2)
$$

And the phase shift is given by:

$$
\Delta \Phi=\mathrm{q} \mathrm{r}
$$

Note that $\mathrm{q} r$ is a scalar product of two vectors, but that for isotropic samples the final result is usually expressed in terms of the norm of q given by eq.(3). The phase shift is thus zero for scattering detected in the forward direction $(\Theta=0)$, which will be of importance below. This is also represented graphically in Figure 7. For the moment, it is noted that a measurable impact of a phase shift is possible if the phase shift is of the order of one (or $\pi$ to be exact). If atoms are close ( $\mathrm{r}$ small), i.e. if one looks at local structures, then $\mathrm{q}$ has to be large in order for the product to be non-zero. On the contrary, if one wishes to study large structures ( $r$ big), then, one has to go to small angles. As said already, typical soft matter systems with sizes greater than the neutron wavelength thus imply measurements at small angles, which gives the name to the technique, SANS.

Finally, the alert reader might have noticed that samples usually contain more than only two atoms. This has two consequences. First, the sum over all the amplitudes of pairs of atoms has to be extended to all pairs in order to get the complete interference. Secondly, individual atoms may be regrouped and described by a density, in a continuum approach. This is justified as the size of the atoms are not probed anyway in small-angle experiments. Then the material is not described any more as a sum of many atoms, but as a continuum of a given density. The scattering length introduced above then becomes a scattering length density:

$$
\rho=\Sigma b_{\mathrm{i}} / \mathrm{V}
$$

This density is usually calculated over a 'small' volume V, where V contains the atoms taken in the sum, each contributing $b_{i}$. In soft matter systems, a typical 'small' volume may be the one of a monomer, or of a homogeneous particle. Heterogeneous systems are then described as a spatial modulation of $\rho(\mathrm{r})$, and in the presence of a matrix of scattering length density $\rho_{\mathrm{m}}$ (which may be a solvent), the contrast $\Delta \rho$ is the origin of small-angle scattering:

$$
\Delta \rho(\mathrm{r})=\rho(\mathrm{r})-\rho_{\mathrm{m}}
$$

Microstructure is thus described by $\Delta \rho(\mathrm{r})$, and in a small angle scattering experiment, the Fourier transform of its auto-correlation function is measured. An intuitive approach to the meaning of this mathematical transformation is presented below. If objects (atoms, molecules, particles...) move, then a time-dependence is introduced, to be picked up by quasi- or inelastic neutron scattering experiments, see [26].

\subsection{Small angle (neutron) scattering}

Note again that textbooks and chapters of previous neutron schools exist [2, 4]. Here we develop an argument allowing an intuitive understanding of form factors. It was explained to 
me some twenty years ago by Loic Auvray, during a long night of SANS measurements, and has helped me ever since. In a small-angle scattering experiment, the intensities coming from the superposition of secondary waves interfering on the detector position are counted. If we are interested in the (only theoretically observable) intensity at zero scattering angle, then the amplitudes of the secondary waves add up, because there is no phase shift as said before. Therefore, the total amplitude is proportional to the number of scattering atoms, possibly weighted by their scattering length density. As the intensity is given by the square of the amplitude, a square of this number arises. One of the two factors is usually converted into the volume fraction, via the product with the number density. The remaining factor counts the number of scattering atoms, and it allows us to recognize that very small angle measurements - like with light scattering -, or better, very low-q measurements, give access to the mass of (independent, uncorrelated) objects, like molecules or particles.

Real measurements are done at finite q-values, and interference must lead to a decrease of the intensity with increasing $\mathrm{q}$, from its maximal value at $\mathrm{q} \rightarrow 0$. For individual, finitesized objects, this is called the Guinier domain, and corresponds to a Gaussian in q:

$$
\mathrm{I}(\mathrm{q})=\mathrm{I}_{0} \exp \left(-\mathrm{q}^{2} \mathrm{R}_{\mathrm{g}}^{2} / 3\right)
$$

Here $\mathrm{R}_{\mathrm{g}}$ denotes the radius of gyration of the object. It is the most important quantity describing the dimension of any object of finite size. It is related (and can be calculated accordingly) to the distribution of mass around the center of mass, giving a higher weight to masses which are located far from the center, just as in the mechanics of rotation (gyration). For known geometries, like surfactant micelles, directly usable forms of this equation will be given below.

The parameter of the low-q decrease with $\mathrm{q}$ is thus given by the total size characterized by $\mathrm{R}_{\mathrm{g}}$. This can be generalized to arbitrary q: if we draw a sphere of diameter $\mathrm{q}^{-1}$ around the particle, then all atoms distant by $r$ within that sphere are closer than $q^{-1}$, leading to $q^{*} r$ being a small number. From eq.(4) we know that the phase shift is thus small, and interference will be constructive, leading to the same counting of atoms (or nuclei) as in the above $q \rightarrow 0$ argument. For particles at larger distances, thus located outside the sphere of diameter $\mathrm{q}^{-1}$, there is no constructive interference any more. For form factors at finite $q$, the mass (or volume) inside a sphere of diameter $\mathrm{q}^{-1}$ gives thus the measured intensity at that q-vector:

$$
\mathrm{I}(\mathrm{q}) \approx \text { mass inside sphere of radius } \mathrm{q}^{-1}
$$

Of course this "equation" can only account for the statistical properties of the mass distribution of an object, which is usually something we are interested in. Once one understands the type of object one observes, one can use more specific geometric form factors, like the ones listed by J.S. Pedersen in ref. [2].

An example of an application of eq.(8) is shown in Figure 8. A cylinder is observed at low and high q, leading to a large and a small sphere of diameter $\mathrm{q}^{-1}$. The volume inside the sphere is given by the length times the cross section $\mathrm{S}$ of the cylinder. The intensity is thus large at small q, and decreases with q. As the length is given by $\mathrm{q}^{-1}$, the final intensity reads in the intermediate scattering regime:

$$
\mathrm{I}(\mathrm{q})=\pi \mathrm{S} \Delta \rho^{2} \Phi \mathrm{q}^{-1}
$$

where $\Delta \rho$ is the contrast of the homogeneous cylinder of volume fraction $\Phi$. The prefactor $\pi$ comes from the exact Fourier transform. If the cylinder is of finite size, then there exists a low q-value corresponding to a very large sphere containing the entire cylinder: the intensity reaches a finite plateau, described by eq.(7), with $\mathrm{I}_{0}$ containing the information of the total cylinder mass, or volume. At high q, one reaches the scattering characteristic of the cross section (see below), and finally the Porod regime of the cylinder surface. Here one may note 
that the $\mathrm{q}^{-4}$ Porod law and its generalization to surface fractals, $\mathrm{q}^{-(6-\mathrm{Df})}$ is easily obtained with the same argument, which is left as an exercise to the reader.
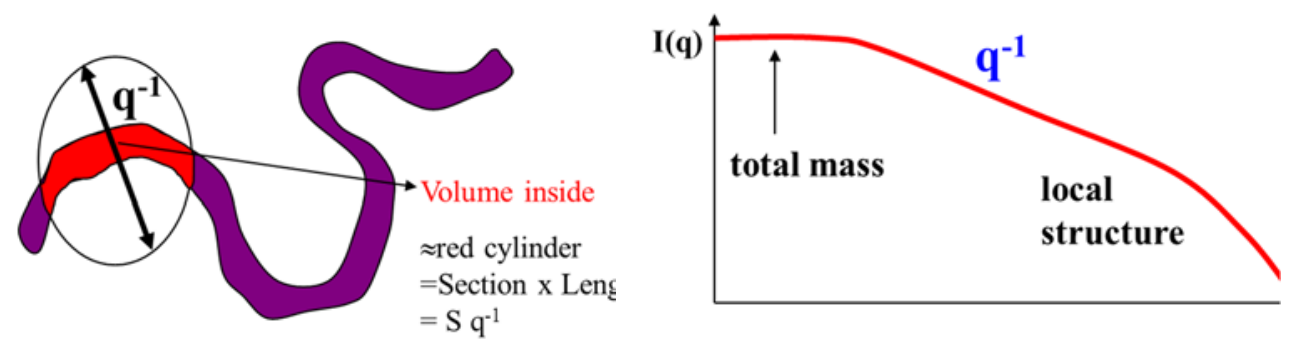

Fig. 8. Example of form factor of a smooth and mostly straight cylinder. The low-q regime gives the total mass and $\mathrm{Rg}$, the intermediate one is calculated using eq.(8) following the sketch on the left, and the high-q domain is given by the Porod law. Undulations of the cylinder as shown on the left would increase the fractal dimension, and thus make the power law steeper.

To summarize, the intermediate scattering regime thus contains information about the spatial distribution of matter. It is closely related to the fractal dimension $\mathrm{d}-$ defined by $\mathrm{N}=$ $(\mathrm{R} / \mathrm{a})^{\mathrm{d}}$ where a is a microscopic scale. Indeed, using this definition, one recognizes that the exponent of minus one in eq.(9) refers to the dimension of one of the cylinder: it is a linear object. If the cylinder were randomly bent, then there would be more mass, the fractal dimension would be higher, and so would the exponent. The latter argument would bring us to the definition of persistence length of wormlike chains from a crossover between fractal regimes, it is also left to the reader. Similarly, the fractal dimension of flat bilayers will lead to $\mathrm{q}^{-2}$ dependence, which is also the case for Gaussian chains. Of course, bilayer scattering (or of any large disk) can be calculated using eq.(8), giving a result similar to eq.(9), but with a prefactor $2 \pi$ and a $1 / \mathrm{q}^{2}$ dependence.

For lack of space, a discussion of structure factors and their peaks cannot be done here. Its thorough treatment necessitates the introduction of concepts of statistical thermomechanics, cf. the chapter by R. Klein in ref. [2]. A simple approach may nonetheless be mentioned, namely dilution lines. It is always beneficial to observe structures as a function of concentration, as scattering peaks move in a particular way depending on the type of order (like liquid crystals, section 3.1), and in particular on its dimensionality. Lamellar phases, e.g., present a one-dimensional order, and the peak position $\mathrm{q}_{0}$ is proportional to the concentration. Deviations appear when the one-dimensional order breaks-down, in particular at low concentrations. Then lamellae are far apart, and can undulate. The measurement of the peak position thus provides a measurement of the bending modulus of the bilayer allowing these undulations, which brings us to the first specific application of scattering, surfactant phases.

\subsection{Small-angle scattering of surfactant phases}

We have seen in section 3 that surfactant molecules self-assemble in geometrically welldefined objects, namely spheres, cylinders, and flat bilayers. The form factor scattering of these objects can be calculated analytically. Note that for anisotropic objects, the orientation needs to be specified, or on the contrary an isotropic average over randomly oriented objects may be appropriate. The Guinier expression for the radius of gyration, eq. (7), can be reformulated for these three geometries, i.e. spheres, cylinders, and flat bilayers, assuming random orientations: 


$$
\begin{aligned}
& \mathrm{I}(\mathrm{q})=\mathrm{V} \Phi \Delta \rho^{2} \exp \left(-\mathrm{q}^{2} \mathrm{R}_{\mathrm{s}}^{2} / 5\right) \\
& \mathrm{I}(\mathrm{q})=\pi \Phi \Delta \rho^{2} \mathrm{~S} \mathrm{q}^{-1} \exp \left(-\mathrm{q}^{2} \mathrm{R}_{\mathrm{c}}^{2} / 4\right) \\
& \mathrm{I}(\mathrm{q})=2 \pi \Phi \Delta \rho^{2} \delta \mathrm{q}-{ }^{2} \exp \left(-\mathrm{q}^{2} \delta^{2} / 12\right)
\end{aligned}
$$

Note that the q-dependent prefactors take the fractal dimension of the objects into account. $R_{s}, R_{c}$, and $\delta$ refer to the geometric radius of the spheres, the cylindrical cross section, and the bilayer thickness, respectively. Fitting the q-dependence of these expressions thus gives both the fractal dimension and the radius or thickness, while the prefactor gives a measure of the amount of scattering material. These two pieces of information should be treated separately: for a homogenous monodisperse spherical mineral particle, e.g., the radius $\mathrm{R}_{\mathrm{s}}$ in eq.(10a) should also give the volume in the prefactor. On the other hand, a polymeric particle might take up solvent, and then extend further into the solution, giving a high "wet" $R_{s}$, whereas the scattering volume is still given by the "dry" particle radius. Comparing the two gives thus an estimation of the solvation. A word of caution about fits might also be necessary: as the intensity is the square of the amplitude, the phase information is lost, and a good fit in itself does not give any guarantee of the geometry. As an example, if the fractal dimensions are unavailable, any high-q fit of the exponentials in eq. (10) might fit the data, or if one does, all do, not allowing one to discriminate between the geometries.

Other geometries exist. Bilayers may bend into spherical objects, called vesicles, see Figure 2. Due to the symmetry, the form factor calculation is straightforward, and has been used to determine electrostatic contributions to bending rigidities, for neat and fuzzy interfaces [97]. As additional information, one might add that some structure factors are particularly suited for surfactant systems. Non-ionic micelles or vesicles are usually well described using the Percus Yevick structure factor [98, 99], and charged ones by RMSA (rescaled mean spherical approximation) [100,101]. Without going into detail, one should at least know that repulsive interactions lead to a scattering peak, of approximate position $2 \pi / \mathrm{D}$, where $\mathrm{D}$ is the typical concentration-dependend center-to-center distance, whereas hard sphere interactions show up only at close contact, i.e. $\mathrm{D}=2 \mathrm{R}$.

\subsection{Small-angle scattering of microemulsions}

Neutrons are particularly suited for the structural characterization of microemulsions as isotopic substitution can be used to highlight either the domains, or the surfactant layer. Again for didactical reasons, we reproduce here the scattering part of a recent review on microemulsions [19]. For simple geometries of domains, like droplets, including non spherical or polydisperse droplets, analytical or quasi-analytical descriptions of the scattered intensity as a function of scattering vector q exist, just like the low-q expressions given in eq.(10). One may also note that a straightforward description of the high-q Porod scattering - if interfaces are well-defined - allows the determination of the specific surface $S / V$, which as already mentioned gives access to the head group area $\sigma$ of the surfactant molecules. Finally, as with any surfactant system, we would like to add that it is possible to describe fuzzy interfaces $[25,102]$.

Bicontinuous structures have been proven to be much harder to describe theoretically, although freeze-fracture electron microscopy gave a clear hint at the structure [103]. Loic Auvray et al have shown early that balanced bicontinuous phases may possess a surfactant layer of zero mean curvature using contrast variation [104]. In this context, one may note the 
discussion of the Kirste-Porod theorem [105] by Auvray and Auroy [106], a theorem which relates the shape of the high-q intensity in Porod presentation $\mathrm{q}^{4} \mathrm{I}(\mathrm{q})$ to the interfacial curvature. The small-angle scattering of interconnected domains has been approached in different ways. First, it was recognized that scattering often displays a peak characterizing a nanometric domain size. It is therefore tempting to divide space in cubes (in 3D), and evaluate the amount of surface between (not-identically filled) neighboring cubes, as a function of the volume fraction $\Phi$ of for instance the water phase. This approach leads to the determination of a dimensionless number $A$, given by the product of the specific surface $S / V$ and the characteristic length $\mathrm{D}$ determined from the peak position:

$$
\mathrm{D} * \mathrm{~S} / \mathrm{V}=\mathrm{A} \Phi(1-\Phi)
$$

In such models, the essential difference between different topologies amounts to different values of the dimensionless number $A$. For cubes, $A=6$. The statistical mechanics of more disordered systems are described by the Talmon-Prager model, which predicts $\mathrm{A}=5.82 \mathrm{using}$ a Voronoi tessellation of space [107-109]. Voronoi-type models are based on a geometrical and random subdivision of space, which is also underlying the family of DOC-models proposed by Zemb et al $[28,31,110,111]$. Obvious difficulties with all of these models are the non physical description of highly bent states at the edges of the cubes or volumes, or at least the non homogeneous distribution of curvature across the samples. Needless to say, fluctuations are entirely absent from such models.

Secondly, analytical models of random mixtures with nanometric domain formation have been proposed, in particular by Debye for inhomogeneous solids [112, 113]. The Debye model for the scattered intensity is simply the Fourier transform of a correlation function $\rho(r)$ with only one characteristic length scale, the domain size $\mathrm{D}: \rho(\mathrm{r}) \approx \exp (-\mathrm{r} / \mathrm{D})$. There is thus no correlation between neighboring domains. In reciprocal space, the intensity reads:

$$
\mathrm{I}(\mathrm{q})=\mathrm{I}_{0} /\left(1+\mathrm{q}^{2} \mathrm{D}^{2}\right)^{2}
$$

If one multiplies out the square in eq.(4), one immediately sees that the dominant high-q term obeys a Porod behavior. The prefactor $\mathrm{I}_{0}$ is related to the product $8 \pi \mathrm{D}^{3} \Delta \rho^{2} \Phi_{1} \Phi_{2}$, where $\Delta \rho$ is the scattering contrast, and $\Phi_{\mathrm{i}}$ are the volume fractions. The surface-to-volume ratio is given by $4 \Phi_{1} \Phi_{2} / \mathrm{D}$. Due to the absence of inter-domain correlations, however, there is no peak predicted by eq.(12), which is linked to the fact that in the expansion of the square, the quadratic and the biquadratic terms have prefactors based on one length only, D. One may note that a similar equation, called the Ornstein-Zernike equation, exists for the description of rapidly decaying thermal fluctuations. Then the correlation function has the Yukawa form $\rho(r) \approx 1 / \mathrm{r} \exp (-\mathrm{r} / \mathrm{D})$, and the intensity is proportional to $1 /\left(1+\mathrm{q}^{2} \mathrm{D}^{2}\right)$. In this case, there is no Porod scattering at high q, and no scattering peak indicative of domain size.

The extension of the Debye scattering law to correlated domains leads to the famous Teubner-Strey formula [114]. The correlation function is now proportional to $1 / \mathrm{r} \sin (2 \pi \mathrm{r} / \mathrm{D})$ $\exp (-\mathrm{r} / \xi)$, and the corresponding intensity may be written as:

$$
\mathrm{I}(\mathrm{q})=\mathrm{I}_{0} /\left(1+\mathrm{B} \mathrm{q}^{2}+\mathrm{C} \mathrm{q}^{4}\right)
$$

Note that fitting eq.(5) to the scattering peaks thus provides the constants B and C, which can be directly transformed into the correlation length $\xi$ and domain size D [115]. Here the prefactors of the $\mathrm{q}^{2}$ and $\mathrm{q}^{4}$ term are now different, which allows the description of the scattering peak. The position of the peak is related to domain size $\mathrm{D}$, whereas its width is related to the correlation length. The more ordered the microemulsion, the higher the correlation length, and thus the thinner the peak. The Teubner-Strey function is extremely successful in describing bicontinous microemulsions. A word of caution should however be added: the presence of a peak is no guarantee of bicontinuity, and indeed it is impossible to 
prove bicontinuity by scattering only, as particulate models might very well work for bicontinuous systems [116]. A peak arising from, say, repelling droplets might be well described by the TS-formula over a limited q-range. It is recommended to always check Porod scattering and dilution lines in order to avoid these traps, and where-ever possible combine with other techniques.

As a last approach to the structure of bicontinous microemulsions, we would like to mention the description of the distribution of oil and water phases using random interfaces as proposed by Safran, Pieruschka, and Marcelja, based on ideas by Cahn and Berk [115, $117,118]$.

\subsection{Small-angle scattering of polymer molecules}

Polymer molecules may have scattering contrast when they are suspended in a (usually deuterated) solvent, or if some deuterated molecules are introduced in melts or networks. The chain conformation in melts has been conjectured to be ideal, i.e. Gaussian, by Flory, and in particular Cotton et al did the first SANS measurements to show that this was actually true [119].

It is recalled that the most useful quantity characterizing individually suspended or embedded macromolecules is the radius of gyration $\mathrm{R}_{\mathrm{g}}$. In section 3 , we were happy to learn that a single distribution function like the Gaussian, eq.(2), gives us the necessary information about the molecules. It has been used by P. Debye to calculate the scattering function of an ideal linear chain [120], which reads:

$$
P_{\text {Debye }}(q)=\frac{2}{q^{4} R_{g}^{4}}\left(\exp \left(-q^{2} R_{g}^{2}\right)-1+q^{2} R_{g}^{2}\right)
$$

As one can see, it contains three pieces of information only: the low-q limit related to chain mass and contrast, the radius of gyration, and the fractal dimension, fixed to two. One may add that a parametrized form of the much more complicated expression for self-avoiding chains has been proposed by Pedersen and Schurtenberger [121, 122].

As an outlook to more complex situations, small-angle scattering may be used to study individual (isotopically labelled) polymer molecule in a network of otherwise identical chains. Moreover, the network can then be macroscopically deformed, and by observing the microscopic chain deformation on a 2D-detector, the transmission of stresses from the macroscopic to the microscopic scale can be investigated [123-126]. Another complex but common situation is the conformation of macromolecules on a surface. If the surface is a nanoparticle, then small-angle scattering can be used to access the polymer structure [81, 127-130]. If on the other hand, the surface is flat, then reflectometry and possibly GISANS are the methods of choice [95, 131-133].

\subsection{Small-angle scattering of colloids and aggregates}

Small-angle scattering of colloids may be discussed separately for form factors $P(q)$ and structure factors $\mathrm{S}(\mathrm{q})$. Ignoring prefactors, the form factors are the intensities measured in dilute suspension, in absence of interactions, where $\mathrm{S}(\mathrm{q})$ equals one. The intuitive picture outlined in sections 4.2 can be used to have an idea of the dominant intermediate-q scattering law. More exact expressions may be obtained by Fourier transforming the contrast function describing the geometry of the particles. For the most important (and easiest) case, spheres of radius RS, one finds: 


$$
P(q)=9 \frac{\left[\sin \left(q R_{S}\right)-q R_{S} \cos \left(q R_{S}\right)\right]^{2}}{\left[q R_{S}\right]^{6}}
$$

Many expressions, often necessitating a numerical integration, have been proposed for different geometries, like cubes for instance (see J. S Pedersen in ref. [2]). As a little exercise, the reader may do the small-q development of eq.(15) and find the Guinier expression, eq. (10a).

The interaction between spheres (or any objects) are described by $\mathrm{S}(\mathrm{q})$. Note that we directly apply two simplifications here, namely monodispersity, and isotropy, which allows us to use the abovementioned structure factors $[98,99,101,134]$ also for liquids or gases of colloids with excluded volume, and possibly electrostatic, or sticky interactions. The scattered intensity is then directly given by the product of $\mathrm{P}(\mathrm{q})$ times $\mathrm{S}(\mathrm{q})$, with a prefactor related to contrast and concentration. A large literature exists on such structure factor calculations based on MC simulations or integral equation theories [135], cf. the chapter by R. Klein in ref. [2], or the one by L. Belloni in ref. [136], respectively, all of which are outside the scope of this introductory chapter.

We would like to discuss shortly another approach to particle interactions, which is often encountered in experiments, mostly unwantedly, but not always. Sticky interactions, e.g. due to van der Waals (dispersion) forces, may induce the formation of aggregates, i.e. particle clustering. The latter may be described by a structure factor of spheres, which may also be looked at as the form factor of aggregates after multiplication by the sphere form factor. Then the thermodynamic problem turns into a geometrical one, because particles now sit on centerto-center distances of two radii. The structure factor of an aggregate of $\mathrm{N}$ monodisperse spheres can be described by the following expression:

$$
S(q)=1+\frac{2}{N} \sum_{i>j}^{N} \frac{\sin \left(q\left(r_{j}-r_{i}\right)\right)}{q\left(r_{j}-r_{I}\right)}
$$

where the Debye average has been used $[120,137]$. The 'secret' of this expression is hidden in the fact that eq.(16) expresses only the isotropically averaged Fourier transform of the center-of-mass positions, but we do not know the latter yet. By building a thermodynamic or geometrical model, one may propose a set of positions [138], and calculate S(q). Its properties are: (i) a high-q limit of one, (ii) a low-q limit of $\mathrm{N}$, i.e. giving the aggregation number from a low-q measurement, (iii) a peak at $2 \pi / 2 \mathrm{R}=\pi / \mathrm{R}$, (iv) possibly a power-law domain at low-q characterizing aggregate fractality, and finally, (v) a correlation hole just before the peak. Note that different ways as compared to eq.(16) to calculate such structure factors exist, like not doing the isotropic average, or using the pair distribution function, their suitability depending on the application.

It immediately follows that one can deduce an estimate of $\mathrm{R}$ and of $\mathrm{N}$ from such measurements. Moreover, one may determine the internal density from the depth of the correlation hole [139]. For polydisperse spheres, and polydisperse aggregates, equivalent relationships can be derived. To finish this discussion, one may also use eq.(16) to proceed with an inverse approach, called reverse Monte Carlo (RMC, see ref. [140]). This allows one to find a distribution of particles in space compatible with the scattered intensity.

Polymer nanocomposites as a typical soft-matter system have been introduced above. The knowledge of aggregate structure is of primary importance, and we have outlined here how one can analyze the corresponding scattering data. Moreover, it is important to characterize not only filler but also chain structure. The latter is possible using the framework of polymer scattering discussed above, with isotopic substitution both to highlight the chain structure, and contrast match the particles. This is called a zero-average contrast (ZAC) measurement. 
To summarize, neutrons are thus perfectly suited for a complete dynamical and structural analysis of nanocomposites.

\section{Conclusion}

We have tried to present a general view on soft matter systems. They are entropy-dominated systems held together by weak interactions, which explore configurational space by Brownian motion. This leads to many fascinating properties of soft matter, like self-assembly of surfactants, forming systems of great morphological variety. They can be understood with the help of a few simple concepts presented in this chapter, like the bending elasticity described by the Helfrich Hamiltonian. Similar approaches have been developed for microemulsions, the statistical mechanics and dynamical properties of polymers, or the random walks or aggregation of colloids. Cold neutrons are perfect probes for such nanoscale structures, and thermally activated transitions or fluctuations, due to their penetration power, their wave-length and energy. In the second part of this chapter, we have tried to outline an intuitive approach to small-angle scattering by typical soft matter structures, and summarized the main tools to extract structural information from scattered intensities.

I thank the organizers of the French-Swedish winter school on neutrons and soft matter, in particular Max Wolff and Fabrice Cousin, for inviting me to Uppsala, as well as for critical reviewing of the present chapter. Some ideas and comments are due to lectures and discussions with S. Egelhaaf (Düsseldorf), A. Ryan (Sheffield), and O. Glatter (Graz).

\section{References}

[1] T. Imae, T. Kanaya, M. Furusaka, N. Torikai, Neutrons in Soft Matter, John Wiley and Sons, Hoboken, New Jersey, 2011.

[2] P. Lindner, Neutrons, X-ray and Light Scattering, North Holland, Elsevier, Amsterdam, 2002.

[3] F. Cousin, I. Grillo, J. Jestin, J. Oberdisse, Neutrons et Matière Molle, EDP Sciences, 2010.

[4] F. Cousin, Small angle neutron scattering, in: M. Ceretti, W. Paulus, M.H. Mathon, C. Ritter (Eds.) Jdn 21 - Neutrons and Materials for Energy, 2015.

[5] C.W. Macosko, Rheology: Principles, Measurements and Applications, Wiley/VCH, New York, 1994.

[6] A. Einstein, Annalen Der Physik, 19 (1906) 371-381.

[7] E.J.W. Verwey, J.T.G. Overbeek, Theory of the stability of lyophobic colloids, Amsterdam: Elsevier., 1948.

[8] J.N. Israelachvili, D.J. Mitchell, B.W. Ninham, Journal of the Chemical Society-Faraday Transactions Ii, 72 (1976) 1525-1568.

[9] D.J. Mitchell, B.W. Ninham, Journal of the Chemical Society-Faraday Transactions Ii, 77 (1981) 601-629.

[10] J. Oberdisse, C. Couve, J. Appell, J.F. Berret, C. Ligoure, G. Porte, Langmuir, 12 (1996) 1212-1218.

[11] P. Alexandridis, U. Olsson, B. Lindman, Abstracts of Papers of the American Chemical Society, 213 (1997) 221-POLY.

[12] P. Alexandridis, U. Olsson, B. Lindman, Langmuir, 13 (1997) 23-34.

[13] P. Alexandridis, U. Olsson, B. Lindman, Langmuir, 14 (1998) 2627-2638.

[14] H. Qiu, M. Caffrey, Biomaterials, 21 (2000) 223-234. 
[15] A. Salonen, S. Guillot, O. Glatter, Langmuir, 23 (2007) 9151-9154.

[16] S. Salentinig, A. Yaghmur, S. Guillot, O. Glatter, Journal of Colloid and Interface Science, 326 (2008) 211-220.

[17] M. Tomsic, S. Guillot, L. Sagalowicz, M.E. Leser, O. Glatter, Langmuir, 25 (2009) 9525-9534.

[18] S. Guillot, S. Salentinig, A. Chemelli, L. Sagalowicz, M.E. Leser, O. Glatter, Langmuir, 26 (2010) 6222-6229.

[19] J. Oberdisse, T. Hellweg, Advances in Colloid and Interface Science (2017) to be published.

[20] W. Helfrich, Zeitschrift Fur Naturforschung C-a Journal of Biosciences, C 28 (1973) 693-703.

[21] G. Porte, Current Opinion in Colloid \& Interface Science, 1 (1996) 345-349.

[22] P. Herve, D. Roux, A.M. Bellocq, F. Nallet, T. Gulikkrzywicki, Journal De Physique Ii, 3 (1993) 1255-1270.

[23] G. Porte, Journal of Physics-Condensed Matter, 4 (1992) 8649-8670.

[24] G. Porte, J. Appell, P. Bassereau, J. Marignan, Journal De Physique, 50 (1989) 13351347.

[25] R. Strey, J. Winkler, L. Magid, J. Phys. Chem., 95 (1991) 7502-7507.

[26] Q. Berrod, K. Lagrené, J. Ollivier, J.-M. Zanotti, Inelastic and Quasi-Elastic Neutron Scattering. Application to Soft-Matter, in: F. Cousin, M. Wolff (Eds.) Neutrons and Soft Matter, EDP Sciences, 2018.

[27] T. Hellweg, D. Langevin, Physical Review E, 57 (1998) 6825-6834.

[28] T.N. Zemb, S.T. Hyde, P.J. Derian, I.S. Barnes, B.W. Ninham, J. Phys. Chem., 91 (1987) 3814-3820.

[29] M. Duvail, J.F. Dufreche, L. Arleth, T. Zemb, Physical Chemistry Chemical Physics, 15 (2013) 7133-7141.

[30] I. Danielsson, B. Lindman, Colloids and Surfaces, 3 (1981) 391-392.

[31] Y. Chevalier, T. Zemb, Reports on Progress in Physics, 53 (1990) 279-371.

[32] L.E. Scriven, Nature, 263 (1976) 123-125.

[33] F. Larche, J. Rouviere, P. Delord, B. Brun, J.L. Dussossoy, Journal De Physique Lettres, 41 (1980) L437-L440.

[34] M. Kahlweit, R. Strey, D. Haase, H. Kunieda, T. Schmeling, B. Faulhaber, M. Borkovec, H.F. Eicke, G. Busse, F. Eggers, T. Funck, H. Richmann, L. Magid, O. Soderman, P. Stilbs, J. Winkler, A. Dittrich, W. Jahn, Journal of Colloid and Interface Science, 118 (1987) 436453.

[35] B. Lindman, K. Shinoda, U. Olsson, D. Anderson, G. Karlstrom, H. Wennerstrom, Colloids and Surfaces, 38 (1989) 205-224.

[36] U. Olsson, K. Shinoda, B. Lindman, J. Phys. Chem., 90 (1986) 4083-4088.

[37] M. Kahlweit, R. Strey, G. Busse, Physical Review E, 47 (1993) 4197-4209.

[38] R. Strey, Colloid Polym. Sci., 272 (1994) 1005-1019.

[39] K. Shinoda, in: K. Shinoda (Ed.) Solvent properties of surfactant solutions, Marcel Dekker, New York, 1967.

[40] K. Shinoda, S.E. Friberg, Emulsions and Solubilization, Wiley-Interscience, New York, 1986.

[41] R. Johannsson, M. Almgren, R. Schomacker, Langmuir, 9 (1993) 1269-1273.

[42] V. Ganesan, G.H. Fredrickson, Journal of Chemical Physics, 113 (2000) 2901-2917.

[43] S. Wellert, H. Imhof, M. Dolle, H.J. Altmann, A. Richardt, T. Hellweg, Colloid Polym. Sci., 286 (2008) 417-426.

[44] S. Vargas-Ruiz, C. Schulreich, A. Kostevic, B. Tiersch, J. Koetz, S. Kakorin, R. von Klitzing, M. Jung, T. Hellweg, S. Wellert, Journal of Colloid and Interface Science, 471 (2016) 118-126. 
[45] M. Doi, S.F. Edwards, The Theory of Polymer Dynamics, Oxford University Press, Oxford, 1986.

[46] M. Doi, Introduction to polymer physics, Oxford Science Publications, Clarendon Press, Oxford, 1996.

[47] B. Frick, D. Richter, Science, 267 (1995) 1939-1945.

[48] B. Ewen, D. Richter, Neutron spin echo investigations on the segmental dynamics of polymers in melts, networks and solutions, in: Neutron Spin Echo Spectroscopy Viscoelasticity Rheology, 1997, pp. 1-129.

[49] A. Arbe, J. Colmenero, D. Richter, M. Monkenbusch, L. Willner, B. Farago, PramanaJournal of Physics, 63 (2004) 33-40.

[50] A.R. Bras, R. Pasquino, T. Koukoulas, G. Tsolou, O. Holderer, A. Radulescu, J. Allgaier, V.G. Mavrantzas, W. Pyckhout-Hintzen, A. Wischnewski, D. Vlassopoulos, D. Richter, Soft Matter, 7 (2011) 11169-11176.

[51] J. Combet, Polyelectrolytes and Small Angle Scattering in: F. Cousin, M. Wolff (Eds.) Neutrons and Soft Matter, EDP Sciences, 2018.

[52] L. Rubatat, Block Copolymer Electrolytes for Fuel Cells and Secondary Batteries, the Small Angle Neutron Scattering Inputs in: F. Cousin, M. Wolff (Eds.) Neutrons and Soft Matter, EDP Sciences, 2018.

[53] S. Mazoyer, L. Cipelletti, L. Ramos, Physical Review Letters, 97 (2006).

[54] G. Brambilla, D. El Masri, M. Pierno, L. Berthier, L. Cipelletti, G. Petekidis, A.B. Schofield, Physical Review Letters, 102 (2009).

[55] E. Michel, M. Filali, R. Aznar, G. Porte, J. Appell, Langmuir, 16 (2000) 8702-8711.

[56] F. Molino, J. Appell, M. Filali, E. Michel, G. Porte, S. Mora, E. Sunyer, Journal of Physics-Condensed Matter, 12 (2000) A491-A498.

[57] E. Michel, J. Appell, F. Molino, J. Kieffer, G. Porte, Journal Of Rheology, 45 (2001) $1465-1477$.

[58] J.F. Berret, G. Cristobal, P. Herve, J. Oberdisse, I. Grillo, European Physical Journal E, 9 (2002) 301-311.

[59] P. Herve, M. Destarac, J.F. Berret, J. Lal, J. Oberdisse, I. Grillo, Europhysics Letters, 58 (2002) 912-918.

[60] J.F. Berret, J. Oberdisse, Abstracts of Papers of the American Chemical Society, 226 (2003) U404-U405.

[61] J.F. Berret, P. Herve, O. Aguerre-Chariol, J. Oberdisse, Journal of Physical Chemistry B, 107 (2003) 8111-8118.

[62] J.-F. Berret, J. Oberdisse, Physica B-Condensed Matter, 350 (2004) 204-206.

[63] J.-F. Berret, B. Vigolo, R. Eng, P. Herve, I. Grillo, L. Yang, Macromolecules, 37 (2004) 4922-4930.

[64] K. Yokota, M. Morvan, J.-F. Berret, J. Oberdisse, Europhys. Lett., 69 (2005) 284-290.

[65] J.-F. Berret, K. Yokota, M. Morvan, R. Schweins, J. Phys. Chem. B, 110 (2006) 1914019146.

[66] J. Fresnais, J.-F. Berret, L. Qi, J.-P. Chapel, J.-C. Castaing, O. Sandre, B. Frka-Petesic,

R. Perzynski, J. Oberdisse, F. Cousin, Phys. Rev. E, 78 (2008) 040401.

[67] I. Hoffmann, S. Prevost, M. Medebach, S. Rogers, N.J. Wagner, M. Gradzielski, Tenside Surfactants Detergents, 48 (2011) 488-494.

[68] L. Chiappisi, I. Hoffmann, M. Gradzielski, Soft Matter, 9 (2013) 3896-3909.

[69] L. Chiappisi, H. Yalcinkaya, V.K. Gopalakrishnan, M. Gradzielski, T. Zemb, Colloid Polym. Sci., 293 (2015) 3131-3143.

[70] H. Frielinghaus, D. Byelov, J. Allgaier, D. Richter, B. Jakobs, T. Sottmann, R. Strey, Applied Physics a-Materials Science \& Processing, 74 (2002) S408-S410.

[71] S. Maccarrone, J. Allgaier, H. Frielinghaus, D. Richter, Langmuir, 30 (2014) 1500-1505. 
[72] M. Abkarian, G. Massiera, L. Berry, M. Roques, C. Braun-Breton, Blood, 117 (2011) 4118-4124.

[73] A. Banc, C. Charbonneau, M. Dahesh, M.S. Appavou, Z. Fu, M.H. Morel, L. Ramos, Soft Matter, 12 (2016) 5340-5352.

[74] M. Dahesh, A. Banc, A. Duri, M.H. Morel, L. Ramos, Food Hydrocolloids, 52 (2016) $1-10$.

[75] B. Wedel, M. Zeiser, T. Hellweg, Zeitschrift Fur Physikalische Chemie-International Journal of Research in Physical Chemistry \& Chemical Physics, 226 (2012) 737-748.

[76] T. Hellweg, Journal of Polymer Science Part B-Polymer Physics, 51 (2013) 1073-1083.

[77] Y. Hertle, T. Hellweg, Journal of Materials Chemistry B, 1 (2013) 5874-5885.

[78] X.L. Wang, M. In, C. Blanc, M. Nobili, A. Stocco, Soft Matter, 11 (2015) 7376-7384.

[79] M. Tatou, Nanocomposites modèles silice-latex: Etude des propriétés rhéologiques et de la structure des charges et des chaînes par Diffusion de Neutrons aux Petits Angles, in: LCVN UMR 5587, University of Montpellier II, Montpellier, 2010.

[80] M. Tatou, A.C. Genix, A. Imaz, J. Forcada, A. Banc, R. Schweins, I. Grillo, J. Oberdisse, Macromolecules, 44 (2011) 9029-9039.

[81] A.C. Genix, M. Tatou, A. Imaz, J. Forcada, R. Schweins, I. Grillo, J. Oberdisse, Macromolecules, 45 (2012) 1663-1675.

[82] A. Banc, A.C. Genix, M. Chirat, C. Dupas, S. Caillol, M. Sztucki, J. Oberdisse, Macromolecules, 47 (2014) 3219-3230.

[83] A. Banc, A.C. Genix, C. Dupas, M. Sztucki, R. Schweins, M.S. Appavou, J. Oberdisse, Macromolecules, 48 (2015) 6596-6605.

[84] G.P. Baeza, A.C. Genix, C. Degrandcourt, L. Petitjean, J. Gummel, R. Schweins, M. Couty, J. Oberdisse, Macromolecules, 46 (2013) 6388-6394.

[85] G.P. Baeza, A.C. Genix, C. Degrandcourt, J. Gummel, A. Mujtaba, K. Saalwächter, T. Thurn-Albrecht, M. Couty, J. Oberdisse, ACS Macro Letters, 3 (2014) 448-452.

[86] G.P. Baeza, A.C. Genix, C. Degrandcourt, L. Petitjean, J. Gummel, M. Couty, J. Oberdisse, Macromolecules, 46 (2013) 317-329.

[87] G.P. Baeza, A.C. Genix, C. Degrandcourt, J. Gummel, M. Couty, J. Oberdisse, Soft Matter, 10 (2014) 6686-6695.

[88] G.P. Baeza, A.-C. Genix, N. Paupy-Peyronnet, C. Degrandcourt, M. Couty, J. Oberdisse, Faraday Discussions, 186 (2016) 295-309

[89] G.P. Baeza, J. Oberdisse, A. Alegria, M. Couty, A.C. Genix, Phys. Chem. Chem. Phys., 17 (2015) 1660-1666.

[90] G.P. Baeza, J. Oberdisse, A. Alegria, K. Saalwaechter, M. Couty, A.-C. Genix, Polymer, 73 (2015) 131-138.

[91] A.C. Genix, G.P. Baeza, J. Oberdisse, European Polymer Journal, 85 (2016) 605-619.

[92] A.-C. Genix, J. Oberdisse, Current Opinion in Colloid \& Interface Science, 20 (2015) 293-303.

[93] T. Ederth, Neutrons for scattering: What they are, where to get them, and how to deal with them, in: F. Cousin, M. Wolff (Eds.) Neutrons and Soft Matter, EDP Science, Les Ulis, 2018.

[94] C. Loupiac, A. Helary, S. Desert, F. Ott, Neutron imaging and tomography: applications in food science in: F. Cousin, M. Wolff (Eds.) Neutrons and Soft Matter, EDP Sciences, 2018.

[95] F. Cousin, A. Chennevière, Neutron reflectivity for Soft Matter, in: F. Cousin, M. Wolff (Eds.) Neutrons and Soft Matter, EDP Sciences, 2018.

[96] M. Wolff, Grazing incidence scattering, in: F. Cousin, M. Wolff (Eds.) Neutrons and Soft Matter, EDP Sciences, 2018.

[97] J. Oberdisse, G. Porte, Physical Review E, 56 (1997) 1965-1975.

[98] J.K. Percus, G.J. Yevick, Physical Review, 110 (1958) 1-13. 
[99] M.S. Wertheim, Physical Review Letters, 10 (1963) 321-\&.

[100] J.B. Hayter, J. Penfold, Molecular Physics, 42 (1981) 109-118.

[101] J.P. Hansen, J.B. Hayter, Molecular Physics, 46 (1982) 651-656.

[102] M. Gradzielski, D. Langevin, L. Magid, R. Strey, J. Phys. Chem., 99 (1995) 1323213238.

[103] W. Jahn, R. Strey, J. Phys. Chem., 92 (1988) 2294-2301.

[104] L. Auvray, J.P. Cotton, R. Ober, C. Taupin, J. Phys. Chem., 88 (1984) 4586-4589.

[105] V.R. Kirste, G. Porod, Kolloid-Zeitschrift and Zeitschrift Fur Polymere, 184 (1962) 1.

[106] L. Auvray, P. Auroy, Scattering by interfaces: variations on Porod's law, in: P. Lindner, T. Zemb (Eds.) Neutron, X-ray, and Light Scattering, 1st edition, North Holland, Amsterdam, 1991.

[107] Y. Talmon, S. Prager, Nature, 267 (1977) 333-335.

[108] Y. Talmon, S. Prager, Journal of Chemical Physics, 76 (1982) 1535-1538.

[109] Y. Talmon, S. Prager, Journal of Chemical Physics, 69 (1978) 2984-2991.

[110] B.W. Ninham, I.S. Barnes, S.T. Hyde, P.J. Derian, T.N. Zemb, Europhysics Letters, 4 (1987) 561-568.

[111] T.N. Zemb, Colloids and Surfaces a-Physicochemical and Engineering Aspects, 129 (1997) 435-454.

[112] P. Debye, A.M. Bueche, Journal of Applied Physics, 20 (1949) 518-525.

[113] P. Debye, H.R. Anderson, H. Brumberger, Journal of Applied Physics, 28 (1957) 679683.

[114] M. Teubner, R. Strey, Journal of Chemical Physics, 87 (1987) 3195-3200.

[115] P. Pieruschka, S.A. Safran, Europhysics Letters, 22 (1993) 625-630.

[116] N. Freiberger, C. Moitzi, L. de Campo, O. Glatter, Journal of Colloid and Interface Science, 312 (2007) 59-67.

[117] P. Pieruschka, S.A. Safran, Journal of Physics-Condensed Matter, 6 (1994) A357A362.

[118] P. Pieruschka, S.A. Safran, Europhysics Letters, 31 (1995) 207-212.

[119] J.P. Cotton, D. Decker, H. Benoit, B. Farnoux, J. Higgins, G. Jannink, R. Ober, C. Picot, J.D. Cloizeau, Macromolecules, 7 (1974) 863-872.

[120] P. Debye, Journal of Physical and Colloid Chemistry, 51 (1947) 18-32.

[121] J.S. Pedersen, M. Laso, P. Schurtenberger, Physical Review E, 54 (1996) R5917R5920.

[122] J.S. Pedersen, P. Schurtenberger, Macromolecules, 29 (1996) 7602-7612.

[123] J. Bastide, J. Herz, F. Boue, Journal De Physique, 46 (1985) 1967-1979.

[124] J. Bastide, F. Boue, Physica A, 140 (1986) 251-260.

[125] F. Boue, B. Farnoux, J. Bastide, A. Lapp, J. Herz, C. Picot, Europhysics Letters, 1 (1986) 637-645.

[126] F. Boue, J. Bastide, M. Buzier, A. Lapp, J. Herz, T.A. Vilgis, Colloid Polym. Sci., 269 (1991) 195-216.

[127] J. Oberdisse, Current Opinion in Colloid \& Interface Science, 12 (2007) 3-8.

[128] G. Carrot, A. El Harrak, J. Oberdisse, J. Jestin, F. Boue, Soft Matter, 2 (2006) 10431047.

[129] J.S. Pedersen, M.C. Gerstenberg, Macromolecules, 29 (1996) 1363-1365.

[130] J.S. Pedersen, C. Svaneborg, Current Opinion in Colloid \& Interface Science, 7 (2002) 158-166.

[131] G. Fragneto, A. Menelle, European Physical Journal Plus, 126 (2011).

[132] G. Fragneto, European Physical Journal-Special Topics, 213 (2012) 327-342.

[133] J. Penfold, R.K. Thomas, Current Opinion in Colloid \& Interface Science, 19 (2014) 198-206.

[134] R.J. Baxter, The Journal of chemical physics, 49 (1968) 2770-2774. 
[135] J.P. Hansen, I.R. McDonald, Theory of Simple Liquids, Academic Press, London, 1986.

[136] L. Belloni, La Diffusion de Neutrons aux Petits Angles: mécanique statistique des liquides et traitement des données, EDP Sciences, 2010.

[137] P. Debye, Annalen Der Physik, 46 (1915) 809-823.

[138] J. Oberdisse, Y. Rharbi, F. Boue, Computational and Theoretical Polymer Science, 10 (2000) 207-217.

[139] A.-C. Genix, J. Oberdisse, Soft Matter, 13 (2017), 8144-8155

[140] J. Oberdisse, P. Hine, W. Pyckhout-Hintzen, Soft Matter, 2 (2007) 476-485. 